\title{
Derivation of 3D Braided Geometry Structures from Braided Symmetry Group
}

\author{
Ma Wensuo", Yang Bingqian and Ren Xiaozhong
}

School of Mechatronics Engineering, Henan University of Science and Technology, Luoyang 471003, China

\begin{abstract}
Considering that there were very a few varieties of 3D braided materials at present, some novel 3D braided geometry structures were derived based on symmetry group theory. Group theory was used for the first time to describe the 3D braided geometry structures was discussed. The whole analyzing procedure from the existing braided geometry structure to the braided symmetry group was described in detail. It is found that because the reflection operation does not exist in some point groups and space groups of braided structure and some odd lattices appeared, the braided symmetry group is not always the same as symmetry groups of crystallographic. The representative volume element of 3D braided geometry structure was deduced from braided space point group, and 3D braided geometry structure was obtained from braided space group. 3D braided geometry structures can not only be classified based on braided group theory, but some of novel 3D braided structures can be deduced through the group's symmetry operators. It is proved that some novel 3D braided processes are feasible. Braided symmetry theory is an effective mathematical method for developing more and rational 3D braided materials.
\end{abstract}

Keywords: 3D braided geometry structure, braided point group, braided space group, novel 3D braided structure.

\section{INTRODUCTION}

The components made of 3D braided composites have been used for the light, heat-resistant structure in the aeronautics and space industry, but the variety of $3 \mathrm{D}$ composites is relatively little at present. Braided composites are a series of materials that their geometry structures are periodic and symmetrical in macroscopic [1-2]. Group theory has become the most useful mathematical tool in modern physics for systematizing the idealized processes dealing with theoretical concepts such as energy, mass, charge, momentum and angular momentum [3]. The same symmetry may occur in various different braided material structures. But they are different from the crystal structure's symmetry and the group theory describing them is relatively self-governed [4], because their inner yarns are intersected each other and formed the complicated spatial meshes. 2D braided geometry structures based on plain group have been analysis [5], 3D braided geometry structures based on space group will be mainly concerned in this paper.

In addition, it is very necessary to research and develop novel 3D braided technology because improvement of existing 3D braided composites' performances may be very difficult, and the variety of 3D braided technology is relatively little at present [4].

\section{BASES OF BRAIDED STRUCTURE GROUP THEORY}

Rotation and Translation Operator. Rotation and translation operator are the most basic operations in the

*Address correspondence to this author at the Mailbox 62, Henan University of Science and Technology, 48 Xiyuan Road, Jianxi District, Luoyang, 471003, China; Tel: +86-379-62330184; Fax: +86-379-62130098; E-mail: mawensuo@126.com,mawensuo@sohu.com symmetry group. The operation relation is denoted with the symbol ' $\{W \mid w\}$ '.

$$
\{W \mid w\} r=W r+w
$$

where the vector ' $r$ ' is a displacement vector of a certain point ' $p$ ' in coordinates. Suppose the coordinate values of ' $p$ ' vary with an operation, ' $r$ ' turns into ' $r$ '. the representation of ' $W$ ' and ' $w$ ' are matrixes, they are

$$
W=\left[\begin{array}{lll}
W_{11} & W_{12} & W_{13} \\
W_{21} & W_{22} & W_{23} \\
W_{31} & W_{32} & W_{33}
\end{array}\right] \quad w=\left[\begin{array}{l}
w_{1} \\
w_{2} \\
w_{3}
\end{array}\right]
$$

The symbol ' $W$ ' is defined as the orthogonal transformation (it contains proper rotation and improper rotation) operator; the symbol ' $w$ ' is translation vector. The symbol ' $\{W \mid w\}$ ' is defined rotation as the translation operator expressing the combination of the rotation and translation operator.

Suppose a yarn in $3 \mathrm{D}$ braided geometry structure is a set of points ' $Q$ ' $\left(p_{i} \in Q\right)$, the vector corresponding to ' $r_{j}$ ' is a displacement vector of a certain point ' $p_{i}$ ' in coordinates, the whole vectors corresponding to the point set ' $Q$ ' are the set ' $R$ ' $\left(r_{j} \in R\right)$. Operated point ' $p_{i}$ ' with the operator of proper rotation and translation ' $\{W \mid w\}$ ' and the vector ' $r$ ' are described in Fig. (1).

Generalized Space Group. Generalized space group (denoted by ' $\Gamma$ ') is defined as a set of the whole point operations, translation operations and their associative operations [6]. 


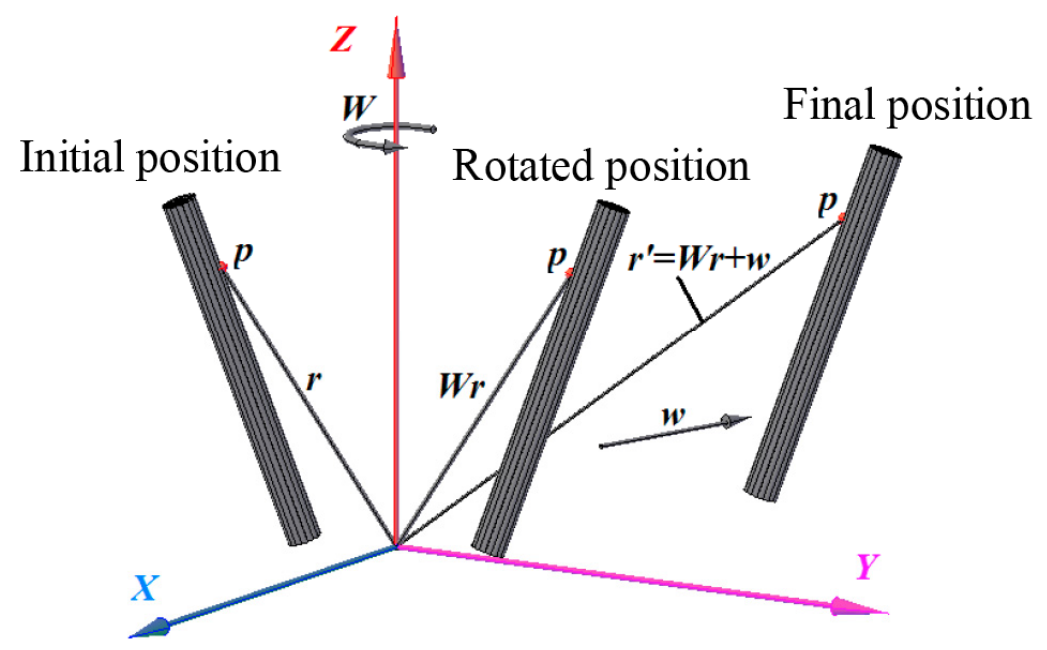

Fig. (1). Operated point ' $p$ ' and corresponds vector' $r$ ' 'with the operator ' $\{W \mid w\}$ '.

(1) The element ' $\{E \mid w\}$ ' of the set is one proper translation operator; ' $\{W \mid 0\}$ ' is one rotation operator; the operator ' $\{E \mid 0\}$ ' is one unit element of generalized space group.

The product of rotation and translation operators is still the same operator

$$
\left\{W \mid w_{1}\right\}\left\{W_{2} \mid w_{2}\right\} r_{1}=\left\{W_{1} W_{2} \mid W_{1} w_{2}+w\right\} \text {. }
$$

Any of operators has their inverse element

$$
\begin{aligned}
& \{W \mid w\}^{-1}=\left\{W^{-1} \mid-W^{-1} w\right\} . \\
& \left\{W_{1} \mid w_{1}\right\}\left[\left\{W_{2} \mid w_{2}\right\}\left\{W_{3} \mid w_{3}\right\}\right]=\left[\left\{W_{1} \mid w_{1}\right\}\left\{W_{2} \mid w_{2}\right\}\right]\left\{W_{3} \mid w_{3}\right\} .
\end{aligned}
$$

Lattice Translation Group. The representative volume element (abbreviated RVE) of 3D braided fabric is a parallelepiped with the least possible volume that can be defined by displacement in non-coplanar directions, ' $a, b$ ' and ' $c$ '. A lattice translation ' $T$ ' is thus determined by the displacement vector [6],

$t_{i}=u_{i} a+v_{i} b+w_{i} c$.

The numbers of ' $u_{i}, v_{i}, w_{i}$ ' are integers, where ' $a, b$ ' and ' $c$ ' are the basis vectors of braided structure lattice.

The multiplication rule of translation operators is the addition of vectors. The periodicity determines that translation through lattice vectors must be considered as the identity operator $\left(t_{i}=0\right)$.

The set ' $T$ ' is lattice translation group, $T=\left\{\left(I, t_{i}\right)\right\}$.

Braided Space Point Group. In general, yarn segments in 3D braided material have a certain space point symmetry. The corresponding symmetry operators may be the inversion ' $\overline{1}$ ' through the central point, or a rotation ' $C_{n}$ ' through the angle ' $2 \pi / n$ ' about $n$-fold axis containing the central point, or any product combination between these operations yielding reflections and rotary reflections. Here ' $n$ ' can take one of the values $1,2,3,4$, and 6 [4].
Lattice translation group ' $T$ ' is a normal subgroup of space group ' $G$ '. These are cosets of space group ' $G$ ' relied on subgroup ' $T$ ' decomposition [6].

Left coset:

$G=T \bigcup T\left(W_{2} \mid w_{2}\right) \bigcup \ldots \bigcup\left(W_{h} \mid w_{h}\right) T$

Right coset:

$G=T \bigcup\left(W_{2} \mid w_{2}\right) T \bigcup \ldots \bigcup T\left(W_{h} \mid w_{h}\right)$

$T\left(W_{i} \mid w_{i}\right)=\left(W_{i} \mid w_{i}\right) T$

The quotient group of space group ' $G$ ' is a set in which its group elements are any one of cosets, which can be expressed as

$G / T=\left\{T, T\left(W_{2} \mid w_{2}\right), \cdots, T\left(W_{h} \mid w_{h}\right)\right\}$

The quotient group ' $G / T$ ' is isomorphism with point group $P=\left\{1, W_{2}, W_{3}, \cdots, W_{h}\right\}$. Group ' $P$ ' is a set of point operations in the group ' $G / T$ '. The group ' $P$ ' is defined as space point group. Group ' $P$ ' ' is the group which is used to describe the symmetry of $3 \mathrm{D}$ braided geometry structures. It is called braided space point group.

Braided space point group ' $P_{b}$ ' is a set of the group elements in the braided space group' $G$ ' in which the whole translation ' $t_{i}$ ' is zero. Translations ' $t_{i}$ ' contain primitive basis and centered ones ' $w_{l}, w_{z}$ '. The proper reflection operation ' $m(\sigma)$ ' is not contained in the set, because the yarn must be continuous and straighten. It is impossible in fabric that the yarn intersecting patterns (as shown in Fig. 2) are satisfied with the symmetries of the corresponding space point groups. However, these space point groups and space groups may be used for deriving new 3D knitting and space trusses structure.

Where braided space point groups are still marked similar to the crystal symmetry group (e. g. $4 m m, 3 m$, etc.)

Obviously, every point operation of braided space point group must be the one of the rotation operations, and its axis 


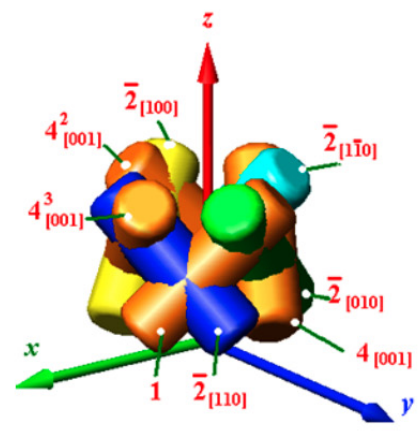

$4 m m$

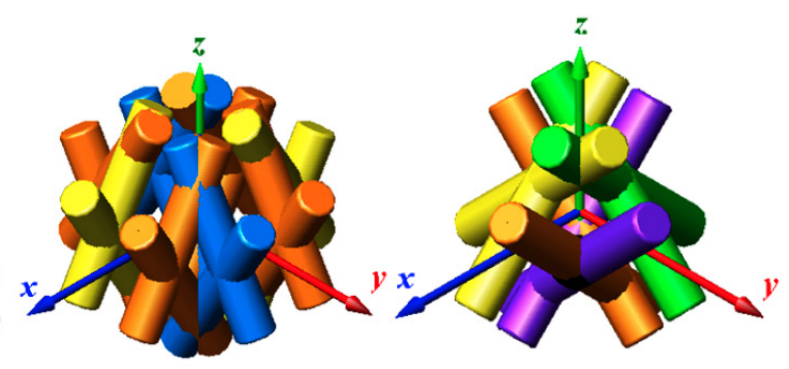

$\frac{4}{m} \frac{2}{m} \frac{2}{m}$ $\overline{4} 2 m$

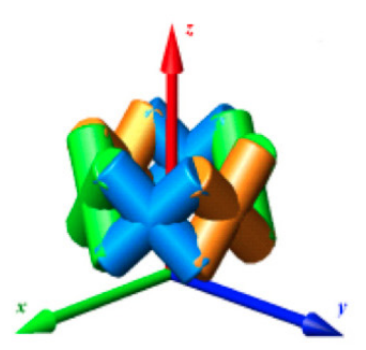

$6 \mathrm{~mm}$

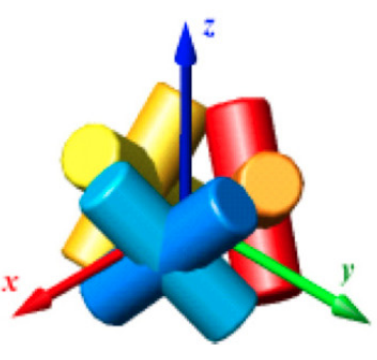

$3 m$

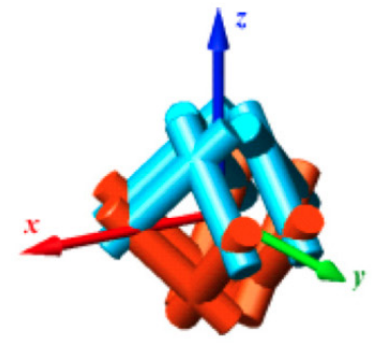

$\overline{3} m$

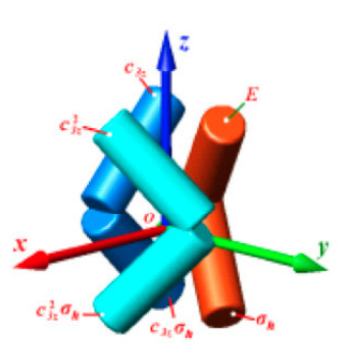

$\overline{6}$

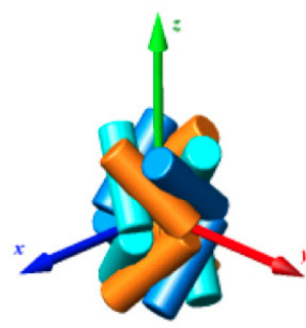

$\frac{6}{m}$

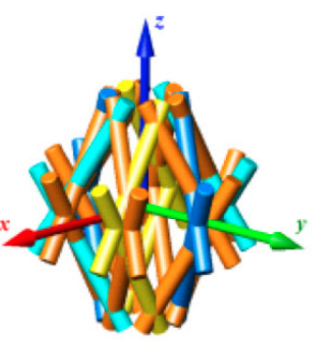

$6 \mathrm{mmm}$

Fig. (2). Abnormality patterns with symmetrical operations of corresponding point groups.

is n-fold, too. Here ' $n$ ' can take one of the values $1,2,3,4$, and 6.

There are fourteen kinds of braided space point group when the aggregate of unidirectional yarn and laminated composites are particularly regarded as 3D braided methods. They are

$$
\begin{aligned}
& C_{1}, C_{2}, C_{3}, C_{4}, C_{6}, S_{2}(\overline{1}), S_{4}(\overline{4}), S_{6}(\overline{3}), D_{2}(222), \\
& D_{3}(32), D_{4}(422), D_{6}(622), T(23), O(432)[6] .
\end{aligned}
$$

The amount of braided point groups is less than the one of crystallographic.

Above all, group ' $O(3)$ ' or ' $T(3)$ ' is subgroup of real affine group ' $\Gamma$ '; braided space group ' $G$ ' is a discrete subgroup of group ' $\Gamma$ '; lattice translation group ' $T$ ' is a discrete subgroup of group ' $\Gamma$ '. Braided space group ' $G$ ' is homomorphism with its quotient group ' $G / T$ '; but the quotient group ' $G / T$ ' is isomorphism with point group ' $P$ '; Braided space point group ' $P$ ' is uniform with point group ' $P$ ' when the proper reflection element ' $m(\sigma)$ ' is not contained in them. That means

$$
\begin{aligned}
& T(3) \leq \Gamma, \quad O(3) \leq \Gamma, \quad G<\Gamma, \quad T<T(3), \quad P \triangleleft G, \\
& G / T \cong P, P_{b}=P(m(\sigma) \notin P)
\end{aligned}
$$

where ' $T(3) \leq \Gamma$ ' is a mark, that ' $T(3)$ ' is a subgroup of ' $\Gamma$ ', but it is not always normal subgroup; ' $G<\Gamma$ ' is a mark, that ' $G$ ' is a normal subgroup of ' $\Gamma$ '; ' $\triangleleft$ 'means homomorphism; ' $\cong$ 'means isomorphism.

Braided Space Group. When the continuous yarn of the braided preform is dispersed, the yarn segment can be expressed by some special symbols or the coordinates and vector describing various states of yarn segment. The existing geometrical structures of 3D braided material are described and rational classified with group theory.

Braided space group ' $G_{b}$ ' is a set of point symmetrical operation ' $W_{b}$ ' (' $W_{b}$ ' is the representation of the group ' $P_{b}$ '), translation operation ' $w$ ' and any product combination ' $\left\{W_{b} \mid w\right\}$ '. The braided geometrical structure is invariable through these transformations.

There are three kinds of operations to describe various states of yarn segment in braided body. The rotation operations ' $W_{b}$ ' of braided space groups only can be the rotation of $1,2,3,4$ or 6 -fold axis [6]. The translation operator ' $w$ ' is divided into two parts. One part is the translation operation ' $w_{l}$ ', which locals the positions of symmetry elements (relied on point, line and plane); the other is the translation operation ' $w_{g}$ ', which is the displacement of translating along glide plane (line) or screw axis. The value of translation operation ' $w_{g}$ ' is especial [6], that means

$w_{g}=k t / n, n=1,2,3,4,6 ; k=1,2,3,4,5 ; 0<k<n$ 


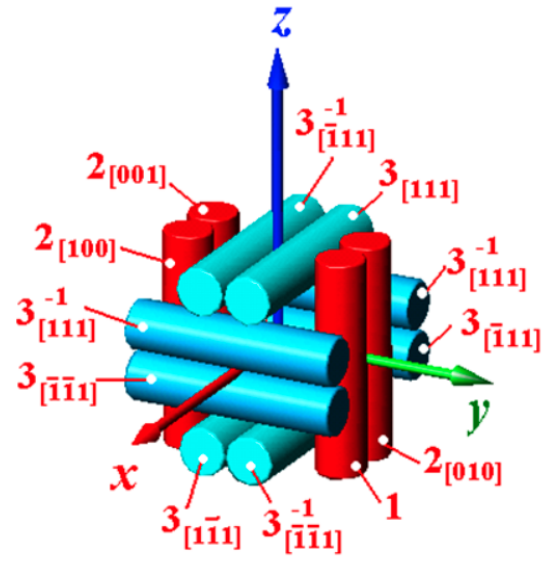

(a) RVE of braided geometry structure corresponding to space point group $23(T)$

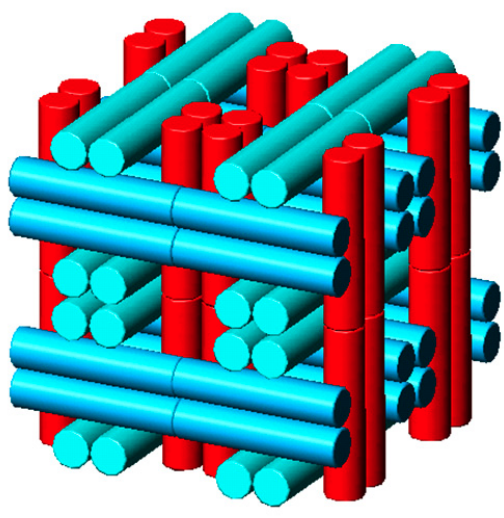

(b) 3D orthogonal braided fabric

Fig. (3). Derivation of the braided space group $P 23\left(T^{1}\right)$ corresponding 3D orthogonal braided geometry structure.

The symbol ' $n$ ' is the fold of screw axis; the symbol ' $k$ ' is the translation times in a cycle ( $\|t\|)$.

Braided space group is divided into two classes, too. One is symmorphic, the other is nonsymmorphic. The combination methods of point group ' $P_{b}$ ' and lattice translation group ' $T$ ' are the same as the ones of crystallographic.

A general element of a braided space group may be written $\left\{W_{b} \mid w\right\}[6]$, where ' $w$ ' is a primitive or nonprimitive translation vector and ' $W_{b}$ ' is a point-group element. In the special case, the braided space group ' $G_{b}$ ' is given by the product of the group ' $T$ ' of primitive translation and braided space point group ' $P_{b}$ '. In symmorphic space groups, the translation vectors ' $w$ ' are always primitive vectors [6]. A symmorphic braided space group is symmorphic and there is not the reflection operation.

The translation vectors ' $w$ ' of the braided space group elements are not always primitive lattice vectors, the group describing 3D braided space geometry structure is denoted as a nonsymmorphic space group. The symmetry operators containing non-primitive lattice translations cannot be obtained for point group elements belong to the symmetry groups. These operators are therefore either screw-rotations or glide-reflections; both ' $W_{b}$ ' and ' $w$ ' are not the elements of the braided space group separately [6].

\section{ANALYSIS OF THE BRAIDED GEOMETRY STRUCTURE}

Analysis Procedure from the Existing Braided Geometry Structures to the Braided Space Group

\section{Braided Geometry Structure of 3D Orthogonal Composites}

In order to find the braided space group of the existing 3D braided geometry structures, a rational RVE with translation symmetry should be found so as to make certain the point symmetry of the braided structure. Translation vector ' $w$ ' is a primitive or non-primitive. The braided space group would be discovered finally.

3D orthogonal composites performance has been studied for a long time [7]. Its structure can be analyzed with corresponding point group ' $T(23)$ ' and space group ' $P 23\left(T^{1}\right)$ ' for the first time.

The RVE of the 3D orthogonal braided fabric is shown in Fig. (3a). Suppose it is an ideal cube, it is certain that the positions of inner yarn are located in the cube.

The lengths of the cubical edge are equal to ' $\mathrm{H}$ '. It is found that the various yarn positions of the RVE are satisfied with whole symmetries of the space point group ' $T(23)$ '. The arbitrary lattice translation ' $t$ ' is thus determined by the displacement vector.

$t=\sum_{i=1}^{3} a_{i} n_{i}$

where ' $a$ ' is a row matrix the elements which are the basis vectors ' $a_{1}, a_{2}$ ' and ' $a_{3}$ ' ( $\left.\left\|a_{i}\right\|=\mathrm{H}\right)$, which are orthogonal to each other. The numbers ' $n_{i}$ ' are all integer. The corresponding lattice is primitive cube.

The corresponding braided space point group is the group ' $T$ (23) '; the braided space group is ' $P 23\left(T^{1}\right)$ '. Its object operated is a yarn segment, which is paralleled to one of cubic edges.

\section{Braided Geometry Structure of 3D 4-Steps Braided Composites}

3D 4-steps braided fabric is an existing method in composites [8-11]. They have been applied in the domains of space flights; its structure is analyzed using corresponding point group and space group. 

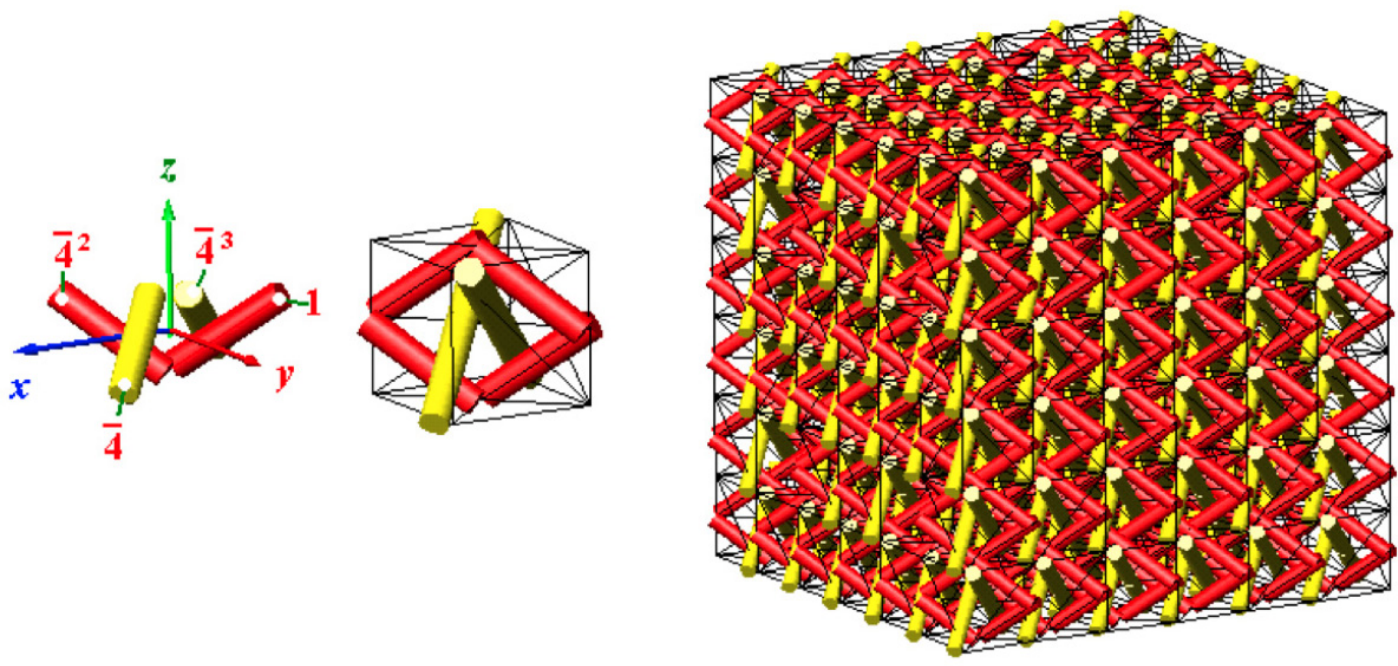

$\begin{array}{lll}\text { (a) RVE corresponds to point group } \overline{4}\left(S_{4}\right) & \text { (b) } 3 \mathrm{D} \text { 4-steps braided fabric }\end{array}$

Fig. (4). Geometry structure of 3D 4-steps braided composites and its braided space group $P 4_{2} 22\left(D_{4}^{5}\right)$.

The process of analysis is shown in Fig. (4). The braided space group ' $P 4_{2} 22\left(D_{4}^{5}\right)$ ' is correspond to the geometry structure of 3D 4-steps braided composites. The detailed course is omitted.

Novel 3D Braided Structure Deduced from Braided Space Group. 3D braided geometry structure can be deduced from space lattice, point group and space group. Investigating octahedral group ' $432(O)$ ', the space group is correspond to the cubic lattice. Symmetrical elements (point, line or surface) contain symmetry axis (line) ' 2 ', ' 3 ' and ' 4 '. There are six branches axle 2-fold, 4 branches axle 3 -fold and 3 branches axle 4-fold, as shown in Fig. (5).

Where ' $c_{n x \bar{y}}^{-1}$ ' means a symmetry operation of ' $n$ ' folds axis which is the angle bisector lying in coordinate ' $x y$ ', plane of second quadrant through zero, superscript '-1' means clockwise rotation.

A yarn segment is put in the position which is the midpoint of the cube arris, and is parallel to the body diagonal, and has the same length as the diagonal at the same time (the contained angle with the ' $x y$ ' coordinate plane is $\left.35^{\circ} 15^{\prime} 52^{\prime \prime}\right)$. The yarn segment is transferred with the symmetry operations of group ' 432 '. Fig. (6a) shows the corresponding equivalent position. The pattern is made up of 12 yarn segments satisfied symmetries of the group ' 432 '. Fig. (6b-d) are the projections towards 2, 3 and 4-fold rotation axis.

The elements of group ' $432(O)$ ' are $1 c_{1}(E), 3 c_{2}\left(c_{2 x}, c_{2 y}, c_{2 z}\right), 6 c_{2}^{\prime}\left(c_{2 x y}, c_{2 x \bar{y}}, c_{2 x z}, c_{2 x \bar{z}}, c_{2 y z}, c_{2 y \bar{z}}\right)$,

$8 c_{3}\left(c_{3 x y z}, c_{3 x y z}^{-1}, c_{3 \bar{x} y \bar{z}}, c_{3 \bar{x} y \bar{z}}^{-1}, c_{3 x \bar{y} \bar{z}}, c_{3 x \bar{y} \bar{z}}^{-1}, c_{3 \bar{x} y \bar{z}}, c_{3 \bar{x} \bar{y} z}^{-1}\right) \quad$ and $6 c_{4}\left(c_{4 x}, c_{4 x}^{-1}, c_{4 y}, c_{4 y}^{-1}, c_{4 z}, c_{4 z}^{-1}\right)[6]$.

3D coordinate ' $x, y, z$ ' is set up, where the direction ' $z$ ' is 4 -fold rotation axis. The new cube just contains all yarn segments as shown in Fig. (6e), which is twice length of the arris of primitive one. There are three branches of screw axis (4-fold) [4].

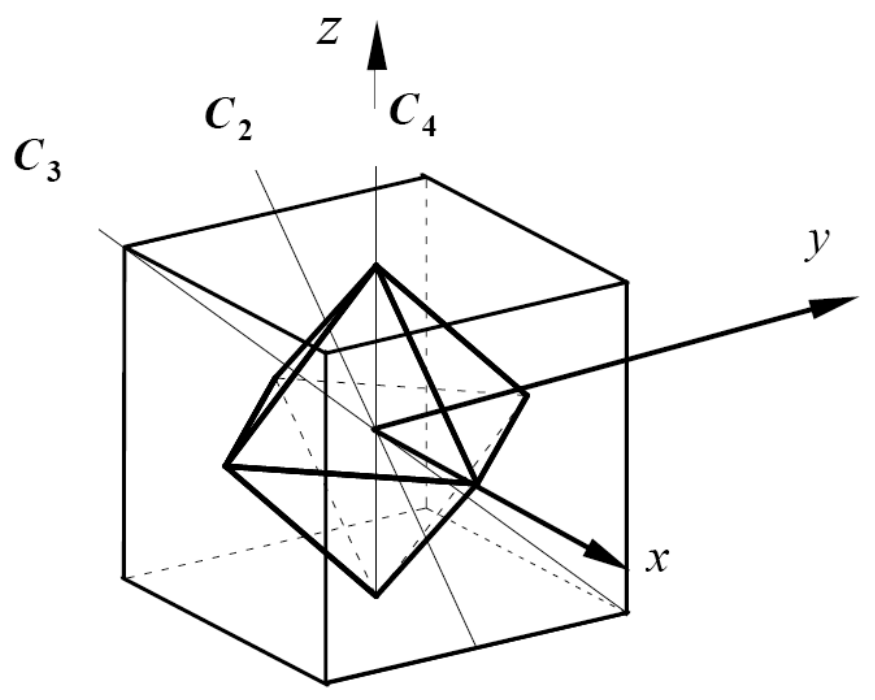

Fig. (5). Symmetry element positions of point group 432.

Corresponding translation group is denoted with $\Gamma=\left\{t \mid t=3\left(n_{1} a+n_{2} b+n_{3} c\right) / 4, n_{1}, n_{2}, n_{3} \quad\right.$ all are integers, $\|a\|=\|b\|=\|c\|=\mathrm{H}\}$; Space group ' $P 4_{3} 32$ ' illustrated in Fig. (6e) is correspond to the 3D braided geometry structure.

Novel 3D braided geometry structure corresponding to space group' $P \overline{3}$ ' has been deduced in a similar way (as shown in Fig. (7)). Fig. (7a) is fabric simulation pattern. Fig. (7b) is fabric sample with rapid prototyping technology. The novel $3 \mathrm{D}$ braided process has been proved to be feasible.

As mentioned above, many novel braided geometry structures have been derived. A more complex "braided" geometry can also be derived from space point group ' 432 ', 

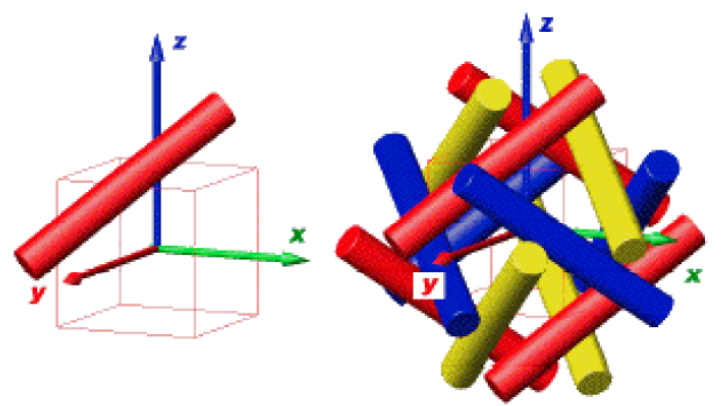

(a) Equivalent pattern of yarn segment with the symmetries of point group 432

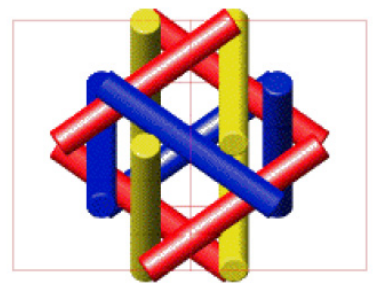

(b) Projection of the pattern in 2- flods axis

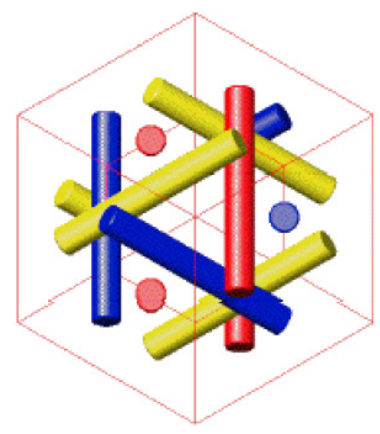

(c) Projection of the pattern in 3-flod axis

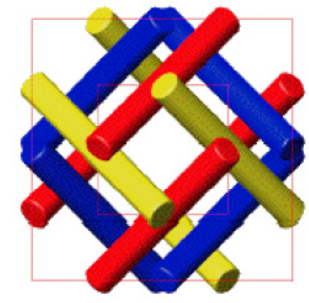

(d) Projection of the pattern in 4-flods axis

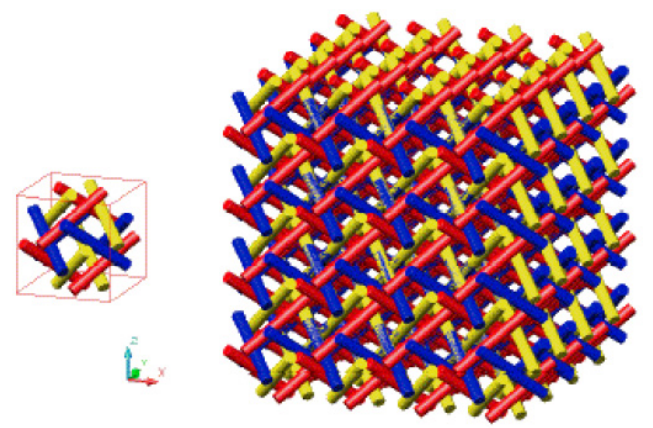

(e) $3 \mathrm{D}$ braided geometry structure of space group $\mathrm{P}_{3} 32$

Fig. (6). Devising procedure of 3D braided geometry structure corresponding to space group $P 4_{3} 32$. 


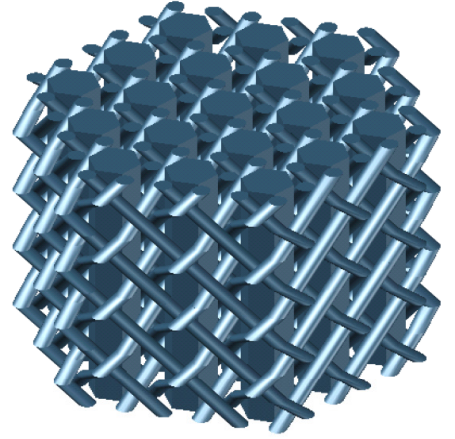

(a)Fabric simulation pattern

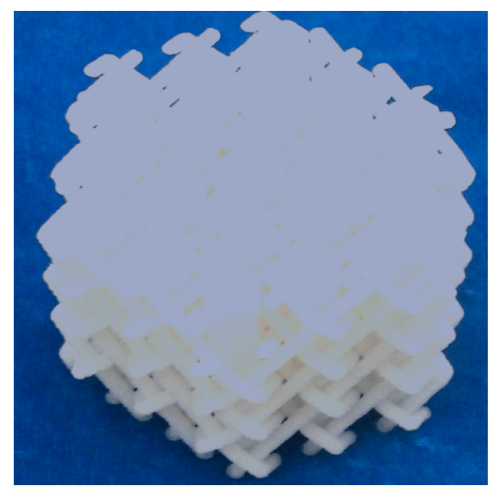

(b) Fabric sample with rapid prototyping technology

Fig. (7). 3D braided geometry structure of space group $P \overline{3}$.

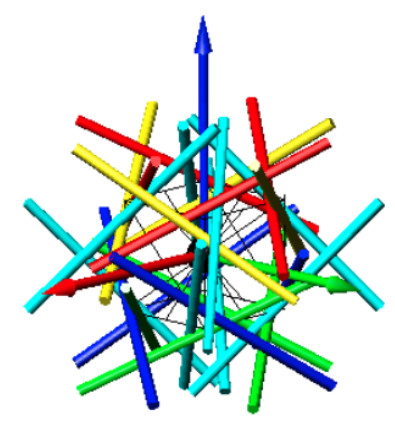

Representative volume element

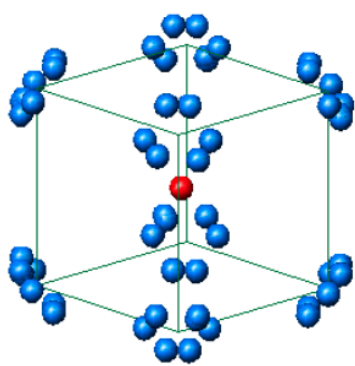

Special lattice

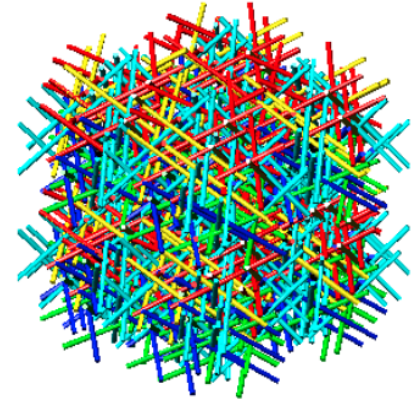

Complex "braided" geometry

Fig. (8). A novel 3D braided geometry structure corresponds to space point group 432 and space group.

and the geometrical structure corresponds to a special lattice, as shown in Fig. (8). As this kind of structure is very complicated, there is no way to change the theoretical model into the practical 3D braided material up to now.

\section{CONCLUSIONS}

In summary, Symmetry groups play an important role in the analysis of braided geometry structures. Analysis of 3D braided geometry structure with point groups and space groups is helpful for finding the potential 3D braided technology. Braided group theory provides theoretical basis for deriving novel braided geometry structures.

\section{ACKNOWLEDGEMENTS}

The subject is supported by doctoral fund of Henan University of Science and Technology and Natural Science of Henan, China. No. 072300440090.

\section{REFERENCES}

[1] Ever J. Barbero, Thomas M. Damiani, Jonathan Trovillion. Micro mechanics of fabric reinforced composites with periodic microstructure. Int J Solids Struct 2005; 42: 2489-504.
[2] Yang Jenn-Ming, Ma Chang-Long, Chou Tsu-Wei. Fiber inclination model of three dimensional textile structural composites. J Compos Mater 1986; 20: 472-84.

[3] Ernest M. Loebl. Group theory and its applications. London. Academic Press Inc. Ltd 1968; 266.

[4] Feng Wei, Ma Wensuo. Group theory analysis of braided geometry structures. Chin Sci Bull 2005; 50: 2529-33.

[5] Ma Wensuo, Feng Wei. Plain group analysis of 2D braided composites geometry. J Univ Sci Technol 2007; 29: 226-31+46.

[6] W. Miller, Jr. Symmetry Groups and their applications. New York. Academic Press 1972; pp. 16-60.

[7] Mouritz AP, Bannister MK, Falzon PJ, et al. Review of applications for advanced three-dimensional fibre textile composites. Composites: Part A 1999; 30: 1445-61.

[8] Li W, Hammad M, El-Shiekn A. Structural analysis of 3-D braided preforms for composites. Part one: The four-step performs. J Textile Inst 1990; 81: 491-514.

[9] Wu DL. Three-cell model and 5D braided structural composites. Compos Sci Technol 1996; 56: 225-33.

[10] Sun HY, Ciao X. Prediction of the mechanical properties of threedimensionally braided composites. Compos Sci Technol 1997; 57: 623-9.

[11] Chen L, Tao XM, Choy CL. On the microstructure of threedimensional braided performs. Compos Sci Technol 1999; 59: 391404. 